\title{
Preditores de sintomas depressivos em crianças e adolescentes institucionalizados ${ }^{*}$
}

Predictors of Depressive Symptoms in Children and Adolescents Institutionalized

Recibido: noviembre 1 de 2008 | Revisado: marzo 16 de 2009 | Aceptado: septiembre 4 de 2009

\author{
JOSIANE LIEBERKNECHT WATHIER ABAID** \\ Débora Dalbosco Dell’Aglio *** \\ Sílvia HeLENA KOLLER ${ }^{* * * *}$ \\ Universidade Federal do Rio Grande do Sul, Brasil
}

Este artigo é parte da dissertação de mestrado da primeira autora junto ao programa de Pós-Graduação em Psicologia da UFRGS. Este estudo teve apoio financeiro da CAPES, FAPERGS e CNPq.

** Programa de Pós-graduação em Psicologia. Rua Ramiro Barcelos, 2600, sala 115. CEP:90035-003. Porto Alegre-RS.josianelieb@yahoo.com.br

* Departamento de Psicologia do Desenvolvimento e da Personalidade. Rua Ramiro Barcelos, 2600/ sala 115 90035-003 - Porto Alegre, RS - Brasil. Correo electrónico: dalbosco@cpovo.net

** Instituto de Psicologia, Cpg Psicologia do Desenvolvimento. Ramiro Barcelos, 2600/104 Santana 90035-003 - Porto Alegre, RS - Brasil - Caixa-Postal: 9001.Correo electrónico: silvia.koller@pq.cnpq.br

\section{RES UMO}

Este estudo investigou eventos de vida estressantes, variáveis psicossociais e preditores de sintomas depressivos em crianças e adolescentes institucionalizados. Participaram 127 crianças e adolescentes de 7 a 16 anos que viviam em abrigos de proteção. Foram realizadas duas aplicações longitudinais do IEEIA e do CDI. Não houve diferença significativa no escore do CDI, e na freqüência de eventos estressores, ao longo do tempo. A análise de regressão linear múltipla permitiu propor um modelo de variáveis preditoras, que, juntas, explicaram $56.7 \%$ da variação nos escores do CDI. A prevenção da ocorrência de eventos relacionados principalmente a conflitos na escola e familiares deve ser enfatizada, bem como a promoção de intervenções junto às famílias em situação de maior vulnerabilidade.

Palavras-chave autoras

Sintomas depressivos; eventos de vida estressantes; institucionalização.

Palavras-chave

Depressão, Eventos estressantes, conflitos interpessoais na adolescência, conflitos interpessoais em crianças.

\section{A B S T R A C T}

This study investigated the stressful life events, psychosocial variables and the predictors of depressive symptoms in 127 participants who were living in shelters. The age range varied from 7 to 16 years old. The CDI and IEEIA were twice administrated. Taking into account the CDI score, the frequency of stressful life events, longitudinal differences were not observed. The multiple linear regression allowed to consider a model of predictors that explain together $56.7 \%$ of the differences on the CDI scores. The prevention of events related mainly to conflicts in the school and within families should be emphasized, as well as the promotion of interventions directed to families in situation of biggest vulnerability.

Key words authors

Depressive Symptoms; Stressful Life Events; Institutionalization.

Key words plus

Depression, Stressful Life Events, Interpersonal Conflict in Adolescence,

Interpersonal Conflict in Children. 
O objetivo deste estudo longitudinal foi investigar eventos de vida estressantes, variáveis psicossociais (número de irmãos, configuração familiar e tempo de institucionalização) e preditores de sintomas depressivos em crianças e adolescentes institucionalizados. O termo depressão foi utilizado para indicar a presença de sintomas depressivos em qualquer nível em crianças e adolescentes, conforme detectado pelo Inventário de Depressão Infantil (CDI; Gouveia, Barbosa, Almeida \& Gaião, 1995; Kovacs, 1983, 1985, 2003). Identificar os fatores de risco preditores de psicopatologia na infância e adolescência, assim como desenvolver formas eficazes de intervir precocemente nestas situações, têm sido alvo de pesquisadores sobre saúde mental (Birmaher et al., 2007; Dell'Aglio \& Hutz, 2004; Masten, 2006). Entre as psicopatologias mais freqüentes na infância e adolescência destaca-se a depressão (Emslie et al., 2004; Zavaschi, Satler, Poester, Vargas, Piazenski, Rohde \& Eizirik, 2002). Apesar de difícil detecção, a hipótese de depressão nestas faixas etárias tem sido cada vez mais considerada em diagnósticos clínicos (Hamilton \& Bridge, 2006; Reppold \& Hutz, 2003). Com relação a episódios depressivos que necessitaram internação, Emslie, Rush, Weinberg e Gullion (1997) verificaram que $47,2 \%$ das crianças e adolescentes internados com quadro de depressão maior apresentaram o retorno dos sintomas até um ano após a alta e $69,2 \%$ nos dois anos seguintes. Esses percentuais caracterizam, segundo os autores, episódios tão ou mais numerosos que os dos adultos.

A complexidade do surgimento da depressão reside, sobretudo, na origem múltipla, de ordem biológica, social e emocional (Bahls, 1999). Em crianças e adolescentes a depressão é caracterizada por sintomas típicos como tristeza, baixa autoestima, fadiga e problemas do sono e da alimentação, além dos atípicos, tais como irritabilidade, agressividade ou tédio (Cole et al., 2002; Marcelli, 1998). Tais sintomas podem mascarar um transtorno depressivo maior, propriamente dito, ou mesmo outros quadros de Eixos I e III (Transtornos Clínicos e Condições Médicas Gerais, respectivamente), conforme o Modelo Multiaxial de Avaliação proposto no DSM-IV-TR (APA, 2000/2002), que prejudicam o desenvolvimento pleno desses jovens (Bahls, 2002). A detecção precoce e prevenção dos sintomas podem evitar danos neuronais e psicossociais, além de reduzir as chances de que crianças e adolescentes desenvolvam outras patologias mais graves ao longo de sua vida (Emslie et al., 2004).

A organização cognitiva das pessoas deprimidas tem sido vista como um processo retroalimentado pelo pessimismo (Bahls, 1999). Pessoas depressivas, como indicam Bandura e Simon (1977), estando pessimistas, facilmente despertam rejeição e assim intensificam sua autodesaprovação, tornandose mais negativas e assim alimentando um ciclo doentio. Abramson, Seligman e Teasdale (1978) enfatizam que o aprendizado da impotência perante os acontecimentos (nomeadamente desamparo aprendido) é responsável pela representação cognitiva de fracasso existente em algumas pessoas deprimidas.

Um dos instrumentos mais usados para avaliar sintomas depressivos é o Inventário de Depressão Infantil (CDI; Gouveia et al., 1995; Kovacs, 1983, 1985, 2003) especialmente utilizado em pesquisas com crianças e adolescentes (Baptista \& Golfeto, 2000; Del Barrio, Olmedo \& Colodrón, 2002). Kovacs $(1985,2003)$ encontrou um Alfa de Cronbach de 0,86 e cinco fatores no seu instrumento: "humor negativo", "problemas interpessoais", "inefetividade", "anedonia" e "auto-estima negativa”. Gouveia et al. (1995), num estudo de adaptação do CDI de 27 itens de Kovacs (1985), encontraram um índice de consistência interna de 0,81 no CDI com 18 itens, que se apresentaram de forma unifatorial. Del Barrio, Olmedo e Colodrón (2002), em um estudo com a versão reduzida do CDI na Espanha obtiveram um alfa de 0,71 e três fatores no instrumento de dez itens: "auto-estima", "anedonia" e "desesperança". Em um estudo de análise fatorial do CDI aplicado em amostras de 7 a 17 anos de algumas cidades do sul do Brasil, Wathier, Dell'Aglio e Bandeira (2008) encontraram um Alfa de Cronbach de 0,85 e três fatores denominados "afetivo-somático", "relação com os outros" e "desempenho". Além destes fatores, as autoras estabeleceram pontos de corte de acordo com a idade e sexo. O CDI apresentou boas 
qualidades psicométricas e permitiu uma avaliação geral da presença e da severidade dos sintomas depressivos como já demonstrado em estudos anteriores (Gouveia et al., 1995; Kovacs, 1983, 1985, 2003; Wathier, Dell'Aglio \& Bandeira, 2008).

Wilde, Kienhorst, Diekstra e Wolters (1992), assim como Prieto e Tavares (2005), afirmaram que a vivência de eventos estressores tem sido associada à depressão, bem como à tentativa ou ideação suicida. Além disso, Merikangas e Angst (1995) destacam que fatores como o aumento da idade, ser do gênero feminino e ter baixo nível socioeconômico podem aumentar a vulnerabilidade das crianças e adolescentes à depressão. Traços de personalidade mais introspectiva e dependente, além de presença de história familiar de depressão e de fatores ambientais desencadeantes, também podem ser fatores de risco para depressão. Por outro lado, o sucesso na vida escolar, o envolvimento em atividades extracurriculares, a competência social, as relações sociais positivas com adultos fora da família, a autopercepção positiva, a competência intelectual, e suportes sociais adequados são características protetoras para que crianças e adolescentes não desenvolvam depressão (Merikangas \& Angst, 1995).

A institucionalização durante a infância e adolescência tem sido apontada como um evento de vida estressante e, portanto, como fator de risco para o desenvolvimento que pode ter como efeito a depressão (Dell'Aglio \& Hutz, 2004; Merikangas \& Angst, 1995). No entanto, conforme o Estatuto da Criança e do Adolescente (1990), o abrigo deve ser uma medida protetiva, excepcional e provisória, que visa, em um primeiro momento, a retirar a criança ou adolescente de alguma situação de risco em que se encontra, com vistas à proteção e garantia dos seus direitos. Assim, a instituição tem a função de proporcionar atenção às diferentes necessidades da criança, como pessoa em desenvolvimento. $\mathrm{O}$ abrigamento institucional deveria, no entanto, ser temporário, até que melhores condições fossem oferecidas às crianças e aos adolescentes, seja em suas famílias de origem ou extensas, ou em casos de adoção.
A institucionalização, durante a infância e a adolescência, mesmo que temporária, tem sido associada a rompimento de vínculos e a situações de violência. Essas experiências podem estar também, relacionadas a sintomas depressivos, conforme já afirmaram Dell'Aglio (2000), Dell'Aglio e Hutz (2003) e Kim e Cicchetti (2006). Além disso, crianças e adolescentes institucionalizados são provenientes de famílias com elevado número de irmãos, caracterizadas por situação de risco, pois aumenta a possibilidade de competição por necessidades básicas e de afeto parental, além de baixa escolaridade (Marteleto, 2002; Yunes \& Szymanski, 2001) e circulação entre diversos cuidadores (Fonseca, 1987, 2002). Sendo assim, objetivou-se identificar preditores de aumento de sintomas depressivos ao longo do tempo, em uma amostra de crianças e adolescentes que vivem sob proteção em abrigos. Também foram investigados eventos de vida estressantes e variáveis psicossociais, como as relacionadas ao número de irmãos, configuração familiar e tempo de institucionalização, entre outras.

\section{Método}

\section{Participantes}

Cento e vinte e sete crianças e adolescentes de ambos os sexos, com idade entre 7 e 16 anos $(M=11,07 ; S D=2,07)$, que residiam em sete instituições de proteção governamentais e duas não governamentais na região metropolitana de Porto Alegre, e que freqüentavam da $1^{\underline{a}}$ a $8^{2}$ série do Ensino Fundamental de escolas públicas compuseram a amostra. O número integral de respondentes em T1 foi 148 e em T2 foi de 127, portanto a perda amostral foi de 21 participantes (14,19\%). Sendo assim, só compuseram a amostra total deste estudo para análises, aqueles que participaram das duas etapas, ou seja, 127 crianças e adolescentes. Destes, 67 eram meninas e 60 meninos que estavam distribuídos da seguinte forma: 20 eram de um abrigo municipal; 81 eram de abrigos estaduais e 26 de instituições não-governamentais. Os participantes 
tinham acesso, devido à inserção destes abrigos na comunidade, aos recursos de escola, centros de lazer, praças e à rede municipal de saúde. Em T2, o tempo de institucionalização dos participantes variou de 6 a 158 meses $(M=43,94$ meses; $S D=36,22)$ e a média de idade dos participantes foi de 12,25 anos $(S D=1,98)$.

\section{Procedimentos e instrumentos}

Este estudo faz parte de um estudo maior, apoiado pelo Pronex/Fapergs e CNPq, que visa a avaliar fatores de risco e de proteção ao desenvolvimento em diferentes contextos. Este projeto foi aprovado pelo Comitê de Ética da UFRGS e as instituições participantes, que mantêm a guarda das crianças e adolescentes, assinaram um Termo de Concordância da Instituição. Os instrumentos foram aplicados individualmente a cada participante, nos próprios abrigos, em horários combinados previamente. Foram realizadas duas a três sessões de aplicação dos instrumentos, conforme o ritmo e a disponibilidade de cada participante.

A partir de listagens de abrigos fornecidas pela Fundação de Proteção Especial do Rio Grande do Sul (FPERGS) e pela Fundação de Assistência Social e Cidadania (FASC), órgãos do governo estadual e municipal, respectivamente, crianças e adolescentes foram selecionados pela idade e escolaridade. A presença de dificuldades que impossibilitassem a compreensão do conteúdo dos instrumentos foi fator de exclusão da amostra.

$\mathrm{Na}$ entrevista inicial foram coletados dados biosociodemográficos, tais como idade, escolaridade, tipo de abrigo, tipo de configuração familiar anterior ao abrigamento, entre outras. Em seguida, foram aplicados individualmente os demais inventários.

O Inventário de Eventos Estressores na Infância e Adolescência (IEEIA), adaptado do Inventário de Eventos Estressores na Adolescência (IEEA; Kristensen, Dell'Aglio, Leon \& D'Incao, 2004), contém 60 itens, que identificam que eventos negativos ocorreram na vida dos participantes e a sua percepção quanto ao impacto de cada um desses eventos. Para cada item, os participantes indicavam a ocorrência/não ocorrência do evento. Em seguida, pontuavam em uma escala Likert de cinco pontos o impacto percebido a cada evento experienciado. $\mathrm{O}$ instrumento apresentou em seu estudo original (Kristensen et al., 2004), uma elevada consistência interna (Alfa de Cronbach =0,92) e neste estudo, o alfa foi de 0,88.

O Inventário de Depressão Infantil (CDI, adaptado para o Brasil por Gouveia et al., 1995) consiste em auto-relato em 27 itens sobre a presença e a severidade dos sintomas depressivos. Para cada item, uma em três opções de respostas (zero a dois) foi escolhida descrevendo o estado dos participantes no período atual. O CDI vem demonstrando características psicométricas adequadas e estudos com amostras brasileiras apresentam consistência interna variando de 0,79 a 0,92 (Dell'Aglio, 2000; Dell'Aglio, Borges \& Santos, 2004; Reppold \& Hutz, 2003). Neste estudo o alfa foi de 0,85 em T1 e 0,89 em T2.

\section{Resultados}

Este estudo atingiu os objetivos de investigar, de forma longitudinal, os eventos de vida estressantes, variáveis psicossociais (número de irmãos, configuração familiar e tempo de institucionalização) e preditores de sintomas depressivos em crianças e adolescentes institucionalizados. Serão descritas a seguir, as configurações familiares dos participantes e as variáveis que se mostraram independentemente associadas à depressão em T2.

Considerando a amostra total, em $89 \%$ dos casos, os pais das crianças e dos adolescentes não viviam juntos e apenas 37,5\% deles conheciam o pai. A maioria $(56,3 \%)$ conhecia a mãe, embora essa já fosse falecida para $31,1 \%$ destes. Aproximadamente sessenta e dois por cento afirmou manter algum tipo de contato com pelo menos um membro da família, em freqüências que variavam desde uma visita semanal a uma visita anual. Antes de ir para o abrigo, $51,7 \%$ da amostra morava com família monoparental. Um Teste $t$ para amostras pareadas identificou a estabilidade do número de eventos e do impacto atribuído aos mesmos em 
T1 e T2. Não foi encontrada diferença significativa, comparando-se T1 e T2, na freqüência de eventos estressores $(t=0,99 ; d f=126 ; p=0,32)$ e no impacto atribuído $(t=-0,78 ; d f=126 ; p=0,44)$. O número de sintomas depressivos também se manteve estável ao longo do tempo, não havendo diferença estatística significativa $(p=0,12)$.

A média do Inventário de Depressão Infantil em T1 foi de 12,46 $(S D=8,14)$ e em T2 foi de 13,42 $(S D=9,38)$. Utilizando as normas estabelecidas por Wathier, Dell'Aglio e Bandeira (2008), baseada em percentis e dividida por sexo e faixa etária, mais de 40 (31,5\% ou mais) crianças e adolescentes do presente estudo encontravam-se com escores de sintomas depressivos clinicamente significativos, tanto em T1 quanto em T2. Análises por sexo e idade revelaram que entre os meninos de 7 a 12 anos, 27,5\% (11) em T1 e 25\% (10) em T2 estavam acima do percentil 85 (ponto de corte $=14$, inclusive). Nessa mesma faixa etária, foram encontradas $31,25 \%$ (15) das meninas com escores clinicamente significativos (maior ou igual a 16) em T1. Em T2, esse percentual elevou-se para 45,83\% (22). $\mathrm{Na}$ faixa etária de 13 a 16 anos, 40\% (8) dos meninos apresentaram escores iguais ou maiores que o percentil 85 (14 pontos ou mais) e esse percentual manteve-se idêntico em T2. Entre as meninas dessa faixa etária (ponto de corte $=18$, inclusive), entretanto, o percentual que era de $31,58 \%$ (6) e baixou para $26,32 \%(5)$.

A média dos eventos estressores obtida através do IEEIA em T1 foi de 26,57 (SD=8,56) e em T2 foi de 25,91 ( $S D=9,33)$. A diferença no total de eventos ocorridos entre T1 e T2 não foi significativa. Entretanto, considerando as diferentes configurações familiares, o número de eventos ocorridos diminuiu entre os participantes cujos pais viviam juntos antes da institucionalização.

A Tabela 1 mostra a distribuição dos participantes nas diferentes configurações familiares e seus escores de freqüência de eventos e de sintomas depressivos em T1 e em T2. Uma Análise de Variância mostrou diferença significativa $\left[F_{(6,126)}=3,05\right.$; $p<0,01]$ na média de eventos entre as diferentes configurações familiares. Uma análise post-hoc (Scheffè) indicou que as crianças e adolescentes com configuração de pai e mãe juntos $(M=18,46$; $S D=7,83)$ possuíam uma média de eventos significativamente menor do que os que viveram aos cuidados de outros familiares antes de vir para o abrigo $(M=30,31 ; S D=12,35)$.

\section{TABELA 1}

Médias e Desvios Padrão do Inventário de Depressão Infantil (CDI) e Freqüência de Eventos Estressantes na Vida em T1 e T2 por Configuração Familiar Anterior ao Abrigamento

\begin{tabular}{|c|c|c|c|c|c|}
\hline \multirow[b]{2}{*}{$\begin{array}{l}\text { Confi- } \\
\text { guração } \\
\text { familiar }\end{array}$} & \multirow[b]{2}{*}{$n$} & \multicolumn{2}{|c|}{ CDI } & \multicolumn{2}{|c|}{ Eventos } \\
\hline & & $\mathrm{T} 1$ & $\mathrm{~T} 2$ & $\mathrm{~T} 1$ & $\mathrm{~T} 2$ \\
\hline Pai ou mãe & 59 & $\begin{array}{l}12,50 \\
(9,13)\end{array}$ & $\begin{array}{l}12,22 \\
(9,84)\end{array}$ & $\begin{array}{l}26,94 \\
(8,33)\end{array}$ & $\begin{array}{l}26,21 \\
(8,75)\end{array}$ \\
\hline $\begin{array}{l}\text { Pai e mãe } \\
\text { juntos }\end{array}$ & 13 & $\begin{array}{c}9,62 \\
(6,79)\end{array}$ & $\begin{array}{l}13,15 \\
(8,68)\end{array}$ & $\begin{array}{c}20,54^{*} \\
(6,37)\end{array}$ & $\begin{array}{l}18,46 \\
(7,83)\end{array}$ \\
\hline $\begin{array}{l}\text { Mãe/ pai ou } \\
\text { padrasto/ma- } \\
\text { drasta }\end{array}$ & 11 & $\begin{array}{l}11,55 \\
(8,03)\end{array}$ & $\begin{array}{l}13,00 \\
(8,60)\end{array}$ & $\begin{array}{l}29,55 \\
(6,27)\end{array}$ & $\begin{array}{l}27,55 \\
(9,11)\end{array}$ \\
\hline $\begin{array}{l}\text { Familiares, } \\
\text { porque os } \\
\text { pais são fale- } \\
\text { cidos }\end{array}$ & 13 & $\begin{array}{l}14,50 \\
(8,39)\end{array}$ & $\begin{array}{c}16,00 \\
(10,86)\end{array}$ & $\begin{array}{l}27,15 \\
(7,03)\end{array}$ & $\begin{array}{l}30,31^{*} \\
(12,35)\end{array}$ \\
\hline $\begin{array}{l}\text { Mãe e/ou pai } \\
\text { adotivos }\end{array}$ & 6 & $\begin{array}{l}13,83 \\
(7,41)\end{array}$ & $\begin{array}{l}15,33 \\
(9,05)\end{array}$ & $\begin{array}{c}30,33 \\
(12,61)\end{array}$ & $\begin{array}{l}28,17 \\
(7,60)\end{array}$ \\
\hline $\begin{array}{l}\text { Familiares, } \\
\text { mas os pais } \\
\text { são vivos }\end{array}$ & 10 & $\begin{array}{l}12,00 \\
(5,64)\end{array}$ & $\begin{array}{l}14,10 \\
(6,38)\end{array}$ & $\begin{array}{c}27,38 \\
(10,82)\end{array}$ & $\begin{array}{l}29,70 \\
(8,99)\end{array}$ \\
\hline $\begin{array}{l}\text { No abrigo } \\
\text { desde bebê }\end{array}$ & 11 & $\begin{array}{l}12,18 \\
(6,95)\end{array}$ & $\begin{array}{c}15,55 \\
(11,36)\end{array}$ & $\begin{array}{l}22,86 \\
(9,37)\end{array}$ & $\begin{array}{c}20,82 \\
(25,84)\end{array}$ \\
\hline $\begin{array}{l}\text { Dado não } \\
\text { coletado } \\
\text { (missings) }\end{array}$ & 4 & $\begin{array}{l}16,33 \\
(4,91)\end{array}$ & $\begin{array}{l}14,00 \\
(4,04)\end{array}$ & $\begin{array}{l}33,33 \\
(6,76)\end{array}$ & $\begin{array}{l}26,67 \\
(5,04)\end{array}$ \\
\hline Total & 127 & $\begin{array}{c}12,42 \\
(8,10)\end{array}$ & $\begin{array}{l}13,42 \\
(9,38)\end{array}$ & $\begin{array}{l}26,60 \\
(8,63)\end{array}$ & $\begin{array}{l}25,91 \\
(9,33)\end{array}$ \\
\hline
\end{tabular}

Nota: $* p<0,01$.

Fonte: elaboração própria.

Comparando a ocorrência de cada um dos eventos estressores na vida, observou-se um aumento na freqüência de 25 eventos (dos 60 existentes) 
conforme apresentado na Tabela 2. Pode-se verificar que os eventos que mais aumentaram entre T1 e T2 foram, em ordem decrescente: "ter algum familiar que usa drogas" (16 ocorrências), "sofrer castigos e punições" (13), "ter problemas com professores" (11) e "tirar notas baixas na escola" (10).

\section{TABELA 2}

Freqüência de Eventos Estressores na Vida Obtidos em T1 e T2 para Itens que Revelaram Aumento $(\mathrm{N}=127)$

\begin{tabular}{|c|c|c|}
\hline Evento & $\mathrm{T} 1$ & $\mathrm{~T} 2$ \\
\hline Ter problemas com professores & 53 & 64 \\
\hline A família ter problemas com polícia & 53 & 55 \\
\hline Discutir com amigos & 95 & 96 \\
\hline Ter problemas com a aparência & 48 & 49 \\
\hline Alguém da família não conseguir emprego & 55 & 59 \\
\hline Mudar de colégio & 98 & 103 \\
\hline Mudar de casa & 86 & 94 \\
\hline Morte de amigo & 36 & 39 \\
\hline Ter familiares doentes ou deficientes & 61 & 62 \\
\hline Ter familiar que usa drogas & 57 & 73 \\
\hline Sofrer castigos e punições & 91 & 104 \\
\hline $\begin{array}{l}\text { Sofrer agressão física ou ameaça de agressão } \\
\text { por parte dos pais }\end{array}$ & 48 & 52 \\
\hline Ser suspenso da escola & 21 & 26 \\
\hline Ser impedido de ver os pais & 42 & 43 \\
\hline Tirar notas baixas & 78 & 88 \\
\hline Usar drogas & 21 & 27 \\
\hline Um dos pais ter que ir morar longe & 42 & 48 \\
\hline Ser estuprado & 10 & 13 \\
\hline Ser rejeitado pelos familiares & 25 & 28 \\
\hline Ser xingado ou ameaçado por professores & 36 & 37 \\
\hline Separação dos pais & 75 & 77 \\
\hline Ser expulso da escola & 8 & 15 \\
\hline Ser expulso sala de aula & 36 & 45 \\
\hline
\end{tabular}

\begin{tabular}{lcc}
\hline \multicolumn{1}{c}{ Evento } & T1 & T2 \\
\hline Sentir-se rejeitado por colegas e amigos & 29 & 32 \\
Sofrer acidente & 39 & 40 \\
\hline
\end{tabular}

Fonte: elaboração própria.

Para identificar variáveis independentemente associadas à depressão ao longo do tempo, foi realizada uma análise de regressão linear múltipla, cuja variável dependente foi o escore total do CDI em T2. Foram introduzidas as seguintes variáveis explicativas: CDI em T1, os 25 eventos que aumentaram a ocorrência em T2 (Tabela 2) e tempo de institucionalização em T2. A Tabela 3 apresenta o resultado da análise com as variáveis que entraram na equação de regressão.

\section{TABELA 3}

Regressão Linear Múltipla: Variáveis Preditoras* do CDI ao Longo do Tempo

\begin{tabular}{lcccc}
\hline \multicolumn{1}{c}{ Variável } & B & Beta & t & p \\
\hline Total do CDI em T1 & 0,78 & 0,67 & 11,05 & $<0,01$ \\
$\begin{array}{l}\text { Ter problemas com pro- } \\
\text { fessores (1) }\end{array}$ & 2,74 & 0,15 & 2,40 & $<0,02$ \\
$\begin{array}{l}\text { Sentir-se rejeitado pelos } \\
\text { colegas e amigos (59) }\end{array}$ & 2,93 & 0,14 & 2,27 & $<0,03$ \\
$\begin{array}{l}\text { Um dos pais ter que mo- } \\
\text { rar longe (48) }\end{array}$ & 2,56 & 0,13 & 2,22 & $<0,03$ \\
Constante & 0,69 & - & 0,57 & 0,57 \\
\hline
\end{tabular}

Nota:*As variáveis estão dispostas conforme o valor de Beta, ou seja, a força de contribuição do item para o escore de CDI em T2. Os números entre parênteses indicam o item do IEEIA.

Fonte: elaboração própria.

Juntas, as quatro variáveis foram responsáveis por $56,7 \%$ da variância no CDI em T2 $\left(R^{2}\right)$. Os coeficientes de regressão padronizados Beta foram positivos, indicando a relação direta entre a variável explicativa e a variável critério. Portanto, tais coeficientes indicaram que o escore obtido na primeira coleta de dados foi fundamental para prever o escore do CDI que a criança ou adolescente 
teria em T2. Ou seja: um nível alto de sintomas depressivos em T1, adicionado das outras três variáveis ("ter problemas com professores", "sentir-se rejeitado pelos colegas e amigos" e "um dos pais ter que morar longe"), aumentou as chances de que ele seria alto em T2. Além dessa variável, ter tido problemas com professores também foi um preditor de que o escore do CDI em T2 estaria mais alto do que em T1. O poder explicativo desse aumento em T2 nos sintomas depressivos foi reforçado pela ocorrência de sentimento de rejeição dos colegas e amigos, assim como morar longe de um dos pais (ex. ser preso ou o abrigo ficar longe da residência da família).

Das 27 variáveis introduzidas na análise, 23 não contribuíram para o modelo explicativo da variação de sintomas depressivos. O sexo, o número de irmãos, o tempo de institucionalização, ou os eventos ter algum familiar que usa drogas, ou ser impedido de ver os pais, por exemplo, não contribuíram diretamente para a equação de regressão.

\section{Discussão}

Este estudo longitudinal verificou a manifestação de sintomas depressivos e sua relação com a ocorrência de eventos estressantes entre a primeira e segunda coleta e algumas variáveis psicossociais (número de irmãos, configuração familiar e tempo de institucionalização). As crianças e adolescentes participantes demonstraram, em conjunto, estabilidade nos sintomas depressivos e no número de eventos estressores ocorridos, no intervalo entre T1 e T2. No entanto, percentual expressivo dos participantes apresentou escores de sintomas depressivos clinicamente significativos já em T1. Também 61 participantes apresentaram aumento no total do CDI em T2, o que sugeriu a importância de se investigar os preditores da depressão, a partir das variáveis apontadas (Escore inicial do CDI, "ter problemas com professores", "sentir-se rejeitado pelos colegas e amigos" e "um dos pais ter que morar longe").

O tempo de institucionalização alto, em contradição com o estabelecido no Estatuto da Criança e do Adolescente (1990), caracterizou marcadamente a amostra, assim como um elevado número de irmãos. Estas duas características observadas na amostra (médias altas de tempo de institucionalização e de número de irmãos) podem ser compreendidas como fatores de risco a que estão expostas as crianças e adolescentes participantes, ainda que não tenham entrado na regressão.

A análise de regressão múltipla mostrou que a variável com maior poder explicativo foi o escore do CDI em T1. A amostra apresentou um elevado percentual de escores de sintomas depressivos clinicamente significativos para cada faixa etária e sexo já em T1. Por exemplo, mais de $45 \%$ das meninas com idade entre 7 e 12 anos apresentaram escores clinicamente significativos. Os estudos de outros pesquisadores que utilizaram o CDI em amostras não clínicas, com o ponto de corte variando de 15 a 20, apresentaram de seis a $24 \%$ de crianças e adolescentes com escores clinicamente significativos (Almqvist et al., 1999; Arnarson, Smari, Einarsdottir \& Jonasdottir, 1994; Bahls, 2002). A comparação entre esses percentuais evidencia o quão alto estavam os escores da amostra do presente estudo. Alguns estudos (Hankin et al., 1998; Lima, 1999; Reppold \& Hutz, 2003) apontam ainda que há uma tendência em encontrar um aumento de escores depressivos principalmente na adolescência em relação à infância. Assim, discute-se a importância de ação terapêutica imediata junto a crianças e adolescentes que apresentarem sintomas depressivos em qualquer nível, tendo em vista que pode haver uma possibilidade de que esses escores aumentem longitudinalmente. Há necessidade, portanto, de um trabalho não só preventivo, mas de intervenção direta quanto aos sintomas depressivos apresentados.

Os eventos que entraram no modelo de regressão, caracterizando-se também como preditores da depressão, foram relacionados a problemas com professores e com pares, além do afastamento de um dos pais. Eventos semelhantes a esses também foram apontados no amplo estudo de revisão de Zavaschi et al. (2002), como uma associação significativa entre trauma por perdas na infância e depressão na vida adulta. Poletto (2007) também 
identificou que escores de depressão, além de afeto negativo, afeto positivo e a freqüência de eventos estressores foram preditores para a satisfação de vida em crianças institucionalizadas em comparação com outras que viviam em situação de pobreza junto às suas famílias. O estudo de Dell'Aglio (2000) salientou que eventos negativos entre crianças e adolescentes institucionalizados envolviam com maior freqüência as categorias "desentendimento com pares" e "privação/afastamento". Este resultado indica perda de qualidade de vida e risco para presença de sintomatologia depressiva. Kristensen et al. (2004) encontraram, entre os eventos mais freqüentes numa amostra de adolescentes, os eventos "ter prova no colégio (84\%)", "discutir com amigos (79\%)" e "morte de algum familiar (que não pais ou irmãos) (73\%). A maior freqüência de eventos estressantes relacionados à escola, amigos e família encontrada nestes estudos reforça os achados do presente estudo, pois essas são categorias de maior ocorrência e que se associam independentemente à depressão.

Ter problemas com professores, apontado com ênfase pelos participantes, reflete a importância do ambiente escolar, que pode ter papel fundamental na manutenção do autoconceito positivo ou negativo, alimentado pelas figuras dos professores (Cubero \& Moreno, 1995). Para Carson e Bittner (1994), experiências estressantes ligadas ao contexto escolar podem levar a fobias, queixas somáticas e episódios depressivos. Dell'Aglio e Hutz (2004) encontraram mais sintomas depressivos e pior desempenho escolar em meninas institucionalizadas. Esses estudos corroboram a urgência de se pensar (e tornar) o ambiente escolar como uma possível fonte de vínculos seguros na vida de pessoas em desenvolvimento.

A importância do relacionamento social com pares também foi evidenciada na presente pesquisa. $\mathrm{O}$ evento "sentir-se rejeitado por colegas e amigos" foi significativo na equação de regressão, indicando que poderia ser considerado um preditor de sintomas depressivos. A importância do grupo durante o desenvolvimento de crianças e principalmente de adolescentes tem sido consensual entre pesquisadores do desenvolvimento. Para as crianças, a convivência social com pares é uma experimentação de como ser aceito, do cumprimento de regras e, conseqüentemente, do desenvolvimento de segurança e auto-estima (Cubero \& Moreno, 1995). Para os adolescentes, a função do grupo é ainda de acolher os conflitos gerados pela transição entre dependência/independência dos adultos (Steinberg, 1999).

A rejeição de um grupo ou de colegas pode, também, se constituir num tipo de bullying, que envolve atos, palavras ou comportamentos prejudiciais, intencionais e repetidos (Middelton-Moz $\&$ Zawadski, 2002, 2007) que são cada vez mais comuns em escolas, entre outros ambientes. Os alvos de bullying são pessoas ou grupos que são prejudicados ou que sofrem as conseqüências de comportamentos agressivos e intencionais, como palavras ofensivas, humilhação, difusão de boatos e agressão física, entre outros. A criança ou adolescente institucionalizado geralmente necessita conviver com o estigma relacionado a esta condição podendo ser vítima de bullying.

Gulassa (2006) destacou que os abrigos refletem a cultura e os preconceitos da cidade onde estão situados. Para ela, nas cidades menores não há tanto problema com a impessoalidade, mas o rótulo de "crianças do abrigo" é também dado com mais facilidade. Esse olhar da sociedade sobre o jovem do contexto institucional torna difícil a tarefa de sair do lugar de abandonado e vitimizado. Também Arpini (2003) e Siqueira e Dell'Aglio (2006) discutem que, ainda que alguns jovens tenham uma imagem positiva do abrigo em suas vidas, sofrem pelo forte estigma social remanescente desta vivência.

Quem sofre agressões de bullying, segundo Middelton-Moz e Zawadski (2002, 2007), costuma não ter esperança quanto às possibilidades de se adequar ao grupo. Ressaltam, ainda, que muitas vítimas passam a ter baixo desempenho escolar, resistem ou recusam-se a ir para a escola, ou abandonam os estudos. Essas características estão diretamente relacionadas aos sintomas depressivos, como confirmam os resultados de Klomek, Marroco, Kleinman, Schonfeld e Gould (2007). Jovens com exposição freqüente ao bullying, tanto como vítima quanto como agressor (bully), tiveram 
alto risco de depressão, ideação suicida e tentativa de suicídio, em relação a jovens que não estiveram envolvidos em comportamento de bullying. No presente estudo (conforme a Tabela 2), "sentir-se rejeitado por colegas e amigos" foi um dos eventos que aumentou sua freqüência ao longo do tempo. Entre os fatores ambientais relacionados ao estresse e à depressão em adolescentes, Steinberg (1999) enfatizou os conflitos e a baixa coesão familiar, além de fraco relacionamento com pares e separação dos pais. Eventos como abuso ou violência na infância, dificuldades de comunicação com os pais, mudanças freqüentes em suas condições de vida, e das pessoas responsáveis por seus cuidados são algumas das evidências apontadas por Prieto e Tavares (2005) como características comuns entre pessoas que cometeram suicídio.

No presente estudo foi identificada uma relação entre a configuração familiar dos participantes e a ocorrência de eventos estressores. A média de eventos estressores ocorridos nesta amostra $(\mathrm{M}=25,91 \mathrm{em} \mathrm{T2})$ é superior ao estudo de Kristensen et al. (2004), que encontraram uma freqüência média de 17,03 eventos em adolescentes que moravam com sua família. Além disso, ter a presença de ambos os pais na família esteve relacionado com menor ocorrência de eventos estressores. Em contrapartida, ficar aos cuidados de outros familiares aumentou a ocorrência de eventos estressores (ver Tabela 1), o que pode indicar a importância do papel protetivo da presença dos pais. Um artigo de revisão sobre preditores de depressão em adolescentes destacou vários estudos abordando a configuração familiar disfuncional. A insatisfação do indivíduo com o apoio familiar e social recebido poderia influenciar os sintomas depressivos em adolescentes (Baptista, Baptista \& Dias, 2001).

O último evento preditor do modelo explicativo do estudo foi "um dos pais ter que morar longe", que pode estar refletindo o perfil das crianças e adolescentes participantes deste estudo, cuja configuração familiar é predominantemente monoparental. Além disso, somente pouco mais da metade dos jovens conheceu sua mãe e bem menos que isso conheceu seu pai, indicando uma configuração familiar que pode estar oferecendo menos recursos de apoio parental e familiar. A configuração monoparental vem sendo descrita na literatura como fator de risco ao desenvolvimento (De Antoni \& Koller, 2000). Contudo, é necessário utilizar o conceito de composição familiar com cautela. Yunes e Szymanski (2001) e Yunes (2003) afirmam ser mais importante verificar o funcionamento da família e as relações ali existentes do que como essas são compostas, embora tais características estejam muitas vezes interligadas. Cárdia e Schiffer (2002), embora descrevam a monoparentalidade como característica que aumenta a vulnerabilidade à violência, não encontraram esse resultado em seu estudo com bairros de São Paulo. Assim, embora o evento "um dos pais ter que morar longe", do modelo preditivo desse estudo, possa ser um indicador da ocorrência de composição familiar monoparental, seria importante poder verificar o funcionamento da família do participante, pois apenas "ser de família monoparental", por si só não qualifica a vulnerabilidade a situações de violência ou à depressão. Indiretamente, a forma como os componentes da rede familiar relacionam-se entre si pode estar favorecendo o surgimento de sintomas depressivos entre essas crianças e adolescentes.

A família tem sido descrita como principal rede de apoio durante a infância e adolescência (Steinberg, 1999). O abrigamento de uma criança ou adolescente, especialmente em casos de extensivos períodos de tempo, pode envolver diretamente a quebra de vínculos e o afastamento da família. Soma-se a isso a constatação, durante o período de coleta de dados e os relatos dos técnicos das instituições, de que poucos jovens mantêm a visitação à família (ou são visitados por esta) durante o período de institucionalização. Assim, ao serem afastados compulsoriamente dos pais, as crianças e adolescentes no abrigo podem perceber este fato como ameaçador para sua identidade e referências de filiação, pertencimento familiar e comunitário (Guará, 2006). Este evento nomeado como "salto para o desconhecido" identifica o afastamento da família, mesmo que esse tenha sido um ambiente ameaçador, como medo de rompimento ou enfraquecimento de laços de parentesco. Trata-se, portanto de um evento de vida estressante que 
pode contribuir para a manifestação de sintomas depressivos.

Apesar das importantes mudanças nas configurações familiares na sociedade de forma geral, Wagner, Ribeiro, Arteche e Bornholdt (1999) salientaram que o tipo de configuração familiar não tem sido associado ao bem-estar dos filhos adolescentes de nível socioeconômico médio e médio-alto. Na qualidade do relacionamento entre os membros da família recai a maior ou menor possibilidade de bem-estar dos adolescentes. Sendo assim, mantêm-se inalterável a função da família de prover o apoio, a proteção e a responsabilidade aos seus filhos. Se a família consegue exercer tal função, conseguirá proteger crianças e adolescentes de eventos de vida estressantes ou ao menos oferecer apoio durante a superação destes (Cowan, Cowan \& Schulz, 1996). Na visão cognitivista, autores como Beck (1997) e Knapp (2002) também salientam que a família é um dos contextos que podem atuar como reforçadores de crenças centrais sejam essas disfuncionais ou não. A composição familiar dos participantes quanto à presença ou não de ambos os genitores, assim como o número de irmãos, possivelmente não entraram na equação de regressão no presente estudo pela forma como foi coletado esse dado. Caso tivéssemos dados sobre como se dava o funcionamento familiar antes da criança ser institucionalizada, poderíamos verificar com mais clareza a relação desta variável com os sintomas depressivos.

Assim, este estudo aponta indicativos de que a vivência de problemas na escola ou na família podem levar a um aumento de sintomas depressivos, de forma cíclica. Os resultados aqui encontrados apontam principalmente para riscos presentes nos diferentes contextos de inserção das crianças e adolescentes (família, pares e escola) e que podem repercutir no ambiente da instituição onde estão abrigados. Desta forma, o olhar contextualizado para o fenômeno torna-se importante, pois não se pode relacionar a manifestação dos sintomas depressivos apenas ao fato dos participantes estarem institucionalizados.

\section{Considerações finais}

Os escores de sintomas depressivos na amostra estudada foram bastante elevados, indicando que, possivelmente, as rupturas familiares e os eventos estressores vivenciados pelas crianças e adolescentes institucionalizados, participantes deste estudo, estão relacionados a repercussões prejudiciais no desenvolvimento. O perfil observado na amostra reflete questões sociais, políticas e econômicas que afetam a constituição e manutenção das famílias, que nem sempre conseguem exercer sua função protetiva. Nestas situações, o abrigo surge com uma função de proteção que deveria ser temporária. No entanto, observa-se que o tempo de institucionalização é longo e que, apesar do constante empenho dos profissionais da instituição em providenciar atendimento psicológico e psiquiátrico, pode-se constatar repercussões negativas, como os sintomas depressivos, entre os participantes institucionalizados.

Além disso, foram observadas médias altas na ocorrência de eventos estressores na amostra, apontando a situação de risco pessoal e social vivenciada por estes jovens. Alguns destes eventos mostraram-se relacionados à depressão, sobretudo eventos relacionados ao contexto escolar, social e familiar. Estes resultados apontam a urgência de trabalhos de intervenção nas escolas e famílias, que possam contribuir para que estes espaços se constituam em redes de apoio social e afetivo. Em jovens de famílias que eram compostas de pai e mãe juntos antes de irem para o abrigo, por exemplo, a freqüência de eventos estressores foi bastante reduzida, confirmando a importância destes para a proteção e desenvolvimento dos filhos.

Quanto aos eventos que aparecem como preditores do escore de sintomas depressivos em T2, poderiam ser amenizados ou evitados com um trabalho preventivo junto à comunidade. Esses eventos podem resultar, indiretamente, de um ciclo de vitimização em que os pais ou outros cuidadores repassam, de forma muitas vezes involuntária, o que aprenderam aos seus filhos, e reproduzem práticas parentais ineficazes e problemáticas (Cecconello, De Antoni \& Koller, 2003). As relações familiares 
disfuncionais também podem estar relacionadas à violência, o que muitas vezes expõe as crianças e adolescentes a acidentes, doenças, discriminação e necessidade de institucionalização.

A identificação da depressão precoce através de instrumentos como o CDI é possível e deve ser feita. Ainda que esse instrumento não tenha o objetivo de diagnosticar transtornos relacionados à depressão, aponta quais os indivíduos necessitam de uma avaliação clínica. Com o screening, pode-se evitar o agravamento do quadro, especialmente em populações que vivem em situações de risco, como em instituições, e melhorar, o mais rapidamente possível, o bem-estar psicológico do jovem em questão. Autores como Kaplan, Sadock e Greeb (1997) destacam, por exemplo, que o tratamento precoce de crianças com Transtorno Depressivo Maior pode evitar o desenvolvimento posterior de Transtorno Bipolar ou Desafiador Opositivo, entre outros. Emslie et al. (2004) também apontam que a detecção precoce e prevenção dos sintomas podem evitar danos neuronais e psicossociais, além de patologias mais graves ao longo da vida.

Dessa forma, sugere-se que sejam criadas ações interventivas junto à comunidade e às instituições com base no modelo proposto a partir dos resultados desse estudo. São necessárias intervenções junto às famílias no sentido de prevenir situações estressoras ao longo do desenvolvimento, que podem, inclusive, ser motivo de abrigamento de crianças e adolescentes, assim como fortalecer o vínculo entre pais e filhos, de forma que estes possam cumprir o papel de proteção e cuidado. Além disso, também é importante a constante capacitação e atualização dos profissionais que atendem crianças e adolescentes em instituições de abrigo. Assim, monitores, educadores e outros funcionários também poderão oferecer a esses jovens um ambiente positivo para o desenvolvimento, evitando conflitos entre pares, e evitando o agravamento de manifestações depressivas.

Uma alternativa para promover os laços afetivos entre as crianças e adolescentes e suas famílias é estimular a visitação destas aos abrigos e viceversa, procurando manter o contato familiar. No presente estudo, ainda que $61,9 \%$ dos participantes tenha afirmado que mantinha algum tipo de contato (não necessariamente em forma de visitação) com pelo menos um membro da família, não se sabe a qualidade desse contato. Num levantamento realizado em 2007 em abrigos de Porto Alegre, mantidos pelo governo estadual (Ministério Público, 2007), apenas $17,78 \%$ das famílias visitavam seus jovens institucionalizados e $16,70 \%$ dos abrigados visitavam suas famílias. Promovendo um contato familiar de qualidade, pode-se favorecer uma maior estabilidade do vínculo afetivo com os familiares na vida das crianças e adolescentes, ainda que este vínculo tenha sido precário até então. Esse processo pode ser mais efetivo se acompanhado por trabalhadores de abrigo, bem como outras pessoas e instituições da comunidade que estimulem a qualidade da relação criança-familiar (Maricondi, 2006).

Sugere-se, ainda, quanto às relações do jovem estabelecidas no contexto escolar, que sejam investigadas em estudos futuros as variáveis que envolvem o fenômeno de bullying. $\mathrm{O}$ contexto escolar pode ser outra possibilidade para desenvolver trabalhos de fortalecimento dos vínculos da criança e do adolescente com familiares, com pares e assim ampliar a rede de apoio desse jovem. Além disso, novos estudos longitudinais com tempo maior de acompanhamento e com amostras clínicas de crianças e adolescentes diagnosticados com depressão também se fazem necessários. Isso poderá ajudar no mapeamento de outros fatores preditores, diferenciando a gravidade dos quadros e a trajetória da manifestação de sintomas de acordo com a história vivencial da criança e do adolescente.

\section{Referências}

Abramson, L. Y., Seligman, M. E. P. \& Teasdale, J. D. (1978). Learned helplessness in humans: Critique and reformulation. Journal of Abnormal Psychology, 87, 49-74.

Almqvist, F., Kumpulainen, K., Ikaeheimo, K., Linna, S-L., Henttonen, I. et al. (1999). Behavioural and emotional symptoms in 8-9-yr-old children. 
European Child and Adolescent Psychiatry, 8, Suppl. 4, 7-16.

American Psychiatric Association. (2000/2002). Manual diagnóstico e estatístico de transtornos mentais (C. Dorneles, Trad., 4 ${ }^{\mathrm{a}}$ ed., texto revisado). Porto Alegre: Artmed.

Arnarson, E. O., Smari, J., Einarsdottir, H. \& Jonasdottir, E. (1994). The prevalence of depressive symptoms in pre-adolescent school children in Iceland. Scandinavian Journal of Behaviour Therapy, 23, 121-130.

Arpini, D. M. (2003). Violência e exclusão: Adolescência em grupos populares. SP: EDUSC.

Bahls, S. C. (1999). Depressão: Uma breve revisão dos fundamentos biológicos e cognitivos. Interação, 3, 49-60.

Bahls, S. C. (2002). Aspectos clínicos da depressão em crianças e adolescentes. Jornal de Pediatria, 78(5), 359-366.

Baptista, C. A. \& Golfeto, J. H. (2000). Prevalência de depressão em escolares de 7 a 14 anos. Revista de Psiquiatria Clínica, 27(5). Retrieved in July 14, 2006, from www.hcnet.usp.br/ipq/revista27(5)/ artigos/art253.htm

Baptista, M. N., Baptista, A. S. D. \& Dias, R. R. (2001). Estrutura e suporte familiar como fatores de risco na depressão de adolescentes. Psicologia: Ciência e Profissão, 21 (2), 52-61.

Beck, J. S. (1997). Terapia cognitiva: Teoria e prática. Porto Alegre: Artes Médicas.

Birmaher, B., Brent, D. A., Kolko, D., Baugher, M., Bridge, J., Holder, D. et al. (2007). Clinical outcome after short-term psychotherapy for adolescents with major depressive disorder. Archives of General Psychiatry, 57, 29-36.

Brasil (1990). Estatuto da criança e do adolescente. Diário Oficial da União. Lei n. 8069, de 13 de julho de 1990. Brasília, DF.

Cardia, N. \& Schiffer, S. (2002). Violência e desigualdade social. Ciência e Cultura, 54(1), 25-31.

Carson, D. K. \& Bittner, M. T. (1994). Temperament and school-aged children's coping abilities and responses to stress. The Journal of Genetic Psychology, 155, 289-302.

Cecconello, A. M., De Antoni, C. \& Koller, S. H. (2003). Práticas educativas, estilos parentais e abuso físico no contexto familiar. Psicologia em Estudo, 8, Número especial, 45-54.

Cole, D. A., Tram, J. M., Martin, J. M., Hoffman, K. B., Ruiz, M. D., Jacquez, F. M. \& Maschman, T. L. (2002). Individual differences in the emergence of depressive symptoms in children and adolescents: A longitudinal investigation of parent and child reports. Journal of Abnormal Psychology, 111(1), 156-165.

Cowan, P. A., Cowan, P. C. \& Schulz, M. S. (1996). Thinking about risk and resilience in families. In E. M. Hetherington \& E. A. Bleachman (Eds.), Stress, coping and resiliency in children and families (pp. 1-38). Nova Jersey: Lawrence Erlbaum Associates

Cubero, R. \& Moreno, M. C. (1995). Relações sociais nos anos escolares: Família, escola, companheiros. In C. Coll, J. Palacios \& A. Marchesi (Eds.), Desenvolvimento psicológico e educação: Psicologia evolutiva (pp. 250-260). Porto Alegre: Artes Médicas.

De Antoni, C. \& Koller, S. H. (2000). Vulnerabilidade e resiliência familiar: um estudo com adolescentes que sofreram maus tratos intrafamiliares. Psico, 31(1), 39-66.

Del Barrio, V., Olmedo, M. L. \& Colodrón, F. (2002). Primera adaptación del CDI-S a población española. Acción Psicológica, 3, 263-272.

Dell'Aglio, D. D. (2000). Oprocesso de coping, institucionalização e eventos de vida em crianças e adolescentes. Unpublished doctoral dissertation, Programa de Pós-Graduação em Psicologia do Desenvolvimento, Universidade Federal do Rio Grande do Sul, Porto Alegre, RS.

Dell'Aglio, D. D. \& Hutz, C. D. (2004). Depressão e desempenho escolar em crianças e adolescentes institucionalizados. Psicologia: Reflexão e Crítica, $17(3), 351-357$.

Dell'Aglio, D. D., \& Hutz, S. H. (2000). Eventos de vida de crianças e adolescentes institucionalizados. Aletheia, 12, 7-20.

Emslie, G. J., Rush, A. J., Weinberg, W. A. \& Gullion, C. M. (1997). Recurrence of major depressive disorder in hospitalized children and adolescents. Journal of the American Academy of Child and Adolescent Psychiatry, 36(6), 785-792. 
Emslie, G. J., Heiligenstein, J. H., Hoog, S. L., Wagner, K. D., Findling, R. L., McCracken, J. T. et al. (2004). Fluoxetine treatment for prevention of relapse of depression in children and adolescents: A double-blind, placebo-controlled study. Journal of the American Academy of Child Eु Adolescent Psychiatry, 43(11), 1397-1405.

Fonseca, C. (1987). O internato do pobre: Febem e a organização doméstica em um grupo porto-alegrense de baixa renda. Temas IMESC, Sociedade, Direito, Saúde, 4(1), 21-39.

Fonseca, C. (2002). Mãe é uma só? Reflexões em torno de alguns casos brasileiros. Psicologia USP, 13(2), 49-68.

Gouveia,V., Barbosa, G., Almeida, H. \& Gaião, A. (1995). Inventário de Depressão Infantil - CDI: Estudo de adaptação com escolares de João Pessoa. Jornal Brasileiro de Psiquiatria, 44, 345-349.

Guará, I. M. F. R. (2006). Abrigo: Comunidade de acolhida e socioeducação. In M. V. Baptista (Ed.), Abrigo: Comunidade de acolhida e socioeducação (pp. 63-72). São Paulo: Instituto Camargo Corrêa.

Gulassa, M. L. C. R. (2006). A fala dos abrigos. In M. V. Baptista (Ed.), Abrigo: Comunidade de acolhida e socieducação (pp. 53-59). São Paulo: Instituto Camargo Corrêa.

Hamilton, J. \& Bridge, J. (2006). Supportive psychotherapy, SSRIs, and MDD. Journal of American Academy of Child EAdolescent Psychiatry, 45 (1), 6-7.

Hankin, B. L., Abramson, L. Y., Moffit, T. E., Silva, P. A., McGee, R. \& Angell, K. E. (1998). Development of depression from preadolescence to young adulthood: emerging gender differences in a 10-year longitudinal study. Journal of Abnormal Psychology, 107, 128-140.

Kaplan, H. I., Sadock, B. J. \& Greeb, J. A. (1997). Transtornos do humor e suicídio. (D. Batista, Trad.). In H. I. Kaplan, B. J. Sadock \& J. A. Greeb (Eds.), Compêndio de Psiquiatria: Ciências do comportamento e psiquiatria clínica (7ª ed., pp. 1039-1045). Porto Alegre: Artes Médicas.

Kim, J. \& Cicchetti, D. (2006). Longitudinal trajectories of self-system and depressive symptoms among maltreated and non-maltreated children. Child Development, 77, 624-639.
Klomek, A. B., Marroco, F., Kleinman, M., Schonfeld, I. S. \& Gould, M. S. (2007). Bullying, depression, and suicidality in adolescents. Journal of the American Academy of Child and Adolescent Psychiatry, 46(1), 40-49.

Knapp, P. (2002). Terapia cognitivo-comportamental no Transtorno de Déficit de Atenção/ Hiperatividade: Manual do terapeuta. Porto Alegre: Artmed.

Kovacs, M. (1983). The Children's Depression Inventory: A self-rated depression scale for school age youngsters. Pittsburg, PA: University of Pittsburgh, School of Medicine.

Kovacs, M. (1985). The Children's Depression Inventory (CDI). Psychopharmacology Bulletin, 21(4), 995-998.

Kovacs, M. (2003). Children's Depression Inventory (CDI): Technical manual update. Toronto: MulthiHealth Systems Inc.

Kristensen, C. H., Dell'Aglio, D. D., Leon, J. S. \& D'Incao, D. B. (2004). Análise da freqüência e do impacto de eventos estressores em uma amostra de adolescentes. Interação, 8(1), 45-55.

Lima, M. S. (1999). Epidemiologia e impacto social. Volume especial Depressão, Revista Brasileira de Psiquiatria, 21, 1-5.

Marcelli, D. (1998). Manual de psicopatologia da infância de Ajuriaguerra (5ª ed.). Porto Alegre: ArtMed.

Maricondi, M. A. (2006). Fortalecer as famílias. In M. V. Baptista (Ed.), Abrigo: Comunidade de acolhida e socieducação (pp. 101-102). São Paulo: Instituto Camargo Corrêa.

Marteleto, L. J. (2002). O papel do tamanho da família na escolaridade dos jovens. Revista Brasileira de Estudos de População, 19(2), s/p.

Masten, A. (2006). Developmental psychopathology: Pathways to the future. International Journal of Behavioral Development, 30(1), 47-54.

Merikangas, K. R. \& Angst, J. (1995). The challenge of depressive disorders in adolescence. In M. Rutter (Ed.), Psychosocial disturbances in young people (pp. 3-6). Londres: Cambrige University Press.

Middelton-Moz, J. \& Zawadski, M. L. (2002/2007). Bullying: Estratégias de sobrevivência para crianças e adultos (R. C. Costa, Trad.). Porto Alegre: Artmed. 
Ministério Público, Rio Grande do Sul. (2007). Rede de abrigos de Porto Alegre: Um olhar sobre a realidade. Porto Alegre: Procuradoria-Geral de Justiça.

Poletto, M. (2007). Contextos ecológicos de promoção de resiliência para crianças e adolescentes em situação de vulnerabilidade. Unpublished master's thesis. Programa de Pós-Graduação em Psicologia, Universidade Federal do Rio Grande do Sul, Porto Alegre, RS.

Prieto, D. \& Tavares, M. (2005). Fatores de risco para suicídio e tentativa de suicídio: incidência, eventos estressores e transtornos mentais. Jornal Brasileiro de Psiquiatria, 54(2), 146-154.

Reppold, C. T. \& Hutz, C. S. (2003). Prevalência de indicadores de depressão entre adolescentes no Rio Grande do Sul. Avaliação Psicológica, 2(2), 175-184.

Siqueira, A. C. \& Dell'Aglio, D. D. (2006). O impacto da institucionalização na infância e na adolescência: Uma revisão de literatura. Psicologia \& Sociedade, 18, 71-80.

Steinberg, L. (1999). Adolescence. Boston: McGraw-Hill.

Wagner, A., Ribeiro, L. de S., Arteche, A. X. \& Bornholdt, E. A. (1999). Configuração familiar e o bem-estar psicológico dos adolescentes. Psicologia: Reflexão e Crítica, 12 (1), 147-156.

Wathier, J. L., Dell'Aglio, D. D. \& Bandeira, D. R. (2008). Análise fatorial do inventário de depressão infantil (CDI) em amostra de jovens brasileiros. Avaliação Psicológica, 7, 75-84.

Wilde, E. J. de, Kienhorst I. C. W. M., Diekstra, R. F. W. \& Wolters, W. H. G. (1992). The relationship between adolescent suicidal behavior and life events in childhood and adolescence. American Journal of Psychiatry, 149 (1), 45-51.

Yunes, M. A. M. \& Szymanski, H. (2001). Resiliência: Noção, conceitos afins e considerações críticas. In J. P. da C. Tavares (Ed.), Resiliência e Educação (pp. 13-42). São Paulo: Cortez.

Yunes, M. A. M. (2003). Psicologia positiva e resiliência: $\bigcirc$ foco no indivíduo e na família. Psicologia em Estudo, 8, 75-84.

Zavaschi, M. L. S., Satler, F., Poester, D., Vargas, C. F., Piazenski, R., Rohde, L. A. P. \& Eizirik, C. L. (2002). Associação entre trauma por perda na infância e depressão na vida adulta. Revista Brasileira de Psiquiatria, 24(4), 189-195. 